\title{
Chest computed tomography findings of 1271 patients with COVID-19 pneumonia and classifications with different age groups: a descriptive study from Istanbul, Turkey
}

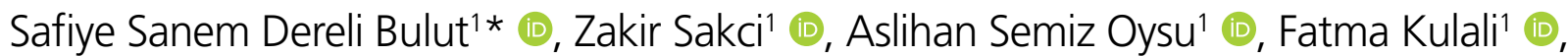 \\ Mehmet Taha Avci ${ }^{1}$, Levent Doganay ${ }^{2}$ (D) Arzu Irvem³ ${ }^{\text {(D), Yasar Bukte }}{ }^{1}$
}

\section{SUMMARY}

OBJECTIVE: The objectives of this study were to describe lung computed tomography findings of patients with COVID-19 diagnosed by real-time reverse transcription polymerase chain reaction test, investigate whether the findings differ regarding age and gender, and evaluate the diagnostic performance of chest computed tomography based on the duration of symptoms at the time of presentation to the hospital. METHODS: From March 11 to May 11, 2020, 1271 consecutive patients (733 males and 538 females) were included in this retrospective, cross-sectional study. Based on age, patients were divided into five separate subgroups. Then based on the duration of symptoms, patients were divided into five separate phases. The presence of lung lesion(s) and their characteristics, distribution patterns, and the presence of concomitant pleural thickening/effusion and other findings (malignancy, metastasis, chronic obstructive pulmonary disease, interstitial lung disease, bronchiectasis, bronchiectasis, cardiomegaly, pericardial effusion) were evaluated by five radiologists independently. RESULTS: The "normal lung computed tomography finding" was the most common chest CT finding (37\%), followed by ground-glass opacity (31\%). Regardless of the shape of the lesion, the distribution features were significant (peripheral, subpleural, and lower lobe distribution) $(p<0.05)$. The presence of pleural thickening posteriorly and adjacent to the lesion was statistically different in groups $1-3$ $(p<0.05)$. Other concomitant pathologies, except pulmonary congestion, did not suppress the typical findings of COVID-19.

CONCLUSION: Chest computed tomography findings were mostly normal in the early phase (P1). Therefore, it may be appropriate to perform the first computed tomography screening of COVID-19 after 6 days to decrease the radiation exposure.

KEYWORDS: COVID-19. X-ray computed tomography. Lung. Pleura.

\section{INTRODUCTION}

A novel coronavirus (SARS-CoV-2) infection, which emerged as "unknown viral pneumonia" in Wuhan, China, in December 2019, spread worldwide in about two months ${ }^{1,2}$. The first case was detected in Turkey on March 11, 2020. After this date, comprehensive diagnostic workup algorithms of possible cases were implemented to ensure the rapid recognition and isolation of patients $s^{3}$. In addition to the reverse transcription polymerase chain reaction (RT-PCR) test, chest computed tomography (CT) was commonly used as a complementary examination ${ }^{4}$.

As reported in the literature, the sensitivity of the RT-PCR test ranges between 60 and $89 \%(5,6)$, which varies based on

\footnotetext{
${ }^{1}$ Health Sciences University, Umraniye Training and Research Hospital, Department of Radiology - Istanbul, Turkey. ${ }^{2}$ Health Sciences University, Umraniye Training and Research Hospital, Genomic Laboratory - Istanbul, Turkey. ${ }^{3}$ Health Sciences University, Umraniye Training and Research Hospital, Department of Microbiology - Istanbul, Turkey. *Corresponding author: ssanembulut@gmail.com Conflicts of interest: the authors declare there are no conflicts of interest. Funding: none. Received on July 26, 2021. Accepted on August 16, 2021.
} 
the location of the sample, as well as the collection of samples, transport, and kit performance ${ }^{5,6}$.

There are many articles in the literature which emphasize the importance of radiological imaging with CT in the diagnosis of the disease ${ }^{4,7-9}$. According to these studies, CT scan shows typical ground-glass opacity (GGO) in the lung, suggesting viral pneumonia. CT findings change rapidly based on the course of the disease. When the RT-PCR test was accepted as a reference, the sensitivity of chest CT was $97 \%$ for the diagnosis of COVID-19 disease ${ }^{8,9}$. For these reasons, knowing the COVID19 CT radiological findings well is extremely important.

This study aimed to document lung CT findings in order of frequency in patients who had a positive RT-PCR test and determine whether lung CT findings vary based on age and gender. In addition, this study also aimed to evaluate the diagnostic performance of chest CT based on the duration of symptoms at the time of presentation to the hospital.

\section{METHODS}

This is a single-center, cross-sectional, retrospective study. It was approved by the ethical committee (54132726-000-8605), and informed consent was received from all the patients. All procedures performed in studies involving human participants were in accordance with the ethical standards of the institutional and/or national research committee and with the 1964 Helsinki Declaration and its later amendments or comparable ethical standards.

All RT-PCR tests were performed at repurposed genomic laboratory with the SARS-CoV-2 detection kit (Coyote Bioscience Co., Ltd.). The primers of the commercial kit were designed to target ORF1ab and $N$ gene. The results of RT-PCR were extracted from the patients' electronic medical records in the Hospital Information System.

\section{Patients}

From March 11 to May 11, 2020, 1500 consecutive patients who underwent chest $\mathrm{CT}$ scan and whose RT-PCR test results positive were included in the study. Patients who had more than 5 days interval between RT-PCR test and CT examination $(\mathrm{n}=51)$, patients with positive RT-PCR test results but no chest CT examination $(\mathrm{n}=152)$, and children below 18 years $(\mathrm{n}=26)$ were excluded from the study.

The mean age of all patient population was 44 years.

Based on age, patients below 44 years and those above 44 years were divided into two groups. Patients above 65 years were considered a separate group due to the high comorbidity (Group 5). Thus, a total of five separate groups were formed.
Patients were divided into five groups as follows: group 1: 18-30 years; group 2: 31-45 years; group 3: 46-55 years; group 4: 56-65 years; and group 5: > 65 years old.

Finally, all the patients included in the study were analyzed based on their complaints with the duration of symptoms. Based on this, chest CT findings were classified radiologically $(0-2$ days=early phase, $3-5$ days=advanced-early phase, 6-10 days=peak phase, and $>11$ days=resolution or recovery phase $)^{4,7}$.

\section{Image analysis}

Image data sets were transferred to a Picture Archiving and Communication System (PACS) workstation for analysis (Centricity ${ }^{\circledR}$ PACS; GE Healthcare, Milwaukee, WI, USA). The images were evaluated by five radiologists independently. All patients were randomly divided into five groups. All observers had over five years of experience in thoracic radiology. Observers evaluated the chest CT findings as follows: type of lesion (GGO and/or consolidation), lung involvement (upper, middle, or lower zone), distribution of the lesion (central, peripheral, or peribronchovascular), and shape of lesion (nodular/round shape, ill-defined borders). In addition, the presence of pleural findings was evaluated (pleural thickening and/or effusion). All the patients were equally shared to five radiologists. Suspected cases were classified in consultation with the most senior radiologist.

\section{Interpretation of the findings}

Based on the previously identified COVID-19 CT findings in the literature ${ }^{10,11}$, the CT impression was collected under four categories:

1. negative for pneumonia,

2. typical appearance for COVID-19,

3. indeterminate appearance for COVID-19, and

4. atypical appearance for COVID-19.

If none of the lesions mentioned above were present, the chest CT was interpreted as normal.

The presence of other existing lung diseases of patients was also recorded as follows: malignancy, metastasis, chronic obstructive pulmonary disease (COPD), interstitial lung disease (ILD), bronchiectasis, cardiomegaly (CMG), and pulmonary congestion, and such patients were included in the category of atypical findings.

\section{Computed tomography protocol}

CT scans were performed in two multidetector scanners (128slice GE Optima CT660 CT scanner, and 16-slice GE Optima CT 520 CT Scanner; GE Healthcare). To minimize motion 
artifacts, the patients were instructed to hold their breath. The CT protocol consisted of tube voltage $=120 \mathrm{kVp}$ (for those below 40 years, tube voltage was $100 \mathrm{kVp}$ ), automatic tube current modulation $(30-70 \mathrm{mAs})$, pitch $=0.99-1.22 \mathrm{~mm}$, matrix $=512 \times 512$, slice thickness $=10 \mathrm{~mm}$, field of view $=350$ $\mathrm{mm} \times 350 \mathrm{~mm}$. All images were then reconstructed with a slice thickness of $0.625-1.250 \mathrm{~mm}$.

\section{Statistical analysis}

All statistical analyses were performed using the Statistical Package for the Social Sciences (SPSS) software (version 22.0; SPSS Inc., Chicago, IL, USA).

The distribution of outcome categories was assessed using the Shapiro-Wilk test. Data are presented as meantstandard deviation, based on the normality of data. And categorical variables were reported as counts and percentages.

When the data distributed normally, a one-way analysis of variance test was performed to check the differences between the groups. If the test showed a significant difference, then the data were further analyzed with a post hoc Tukey's test.

A $p<0.05$ was considered statistically significant.

\section{RESULTS}

\section{Patients and demographic findings}

A total of 1271 patients ( 733 males and 538 females) who met the study criteria were included.

Group 2 was the majority of our patient population with a ratio of $34 \%$. This was followed by groups $1,3,5$, and 4 , in order of frequency $(24,20,11$ and $0.9 \%$, respectively).

The mean interval between the RT-PCR test and the first CT scan was $0.97 \pm 1.83$ days.

The most common symptom at the time of presentation to the hospital was cough in all groups. The second most common symptom was fever. It was also noteworthy that the frequency of hyposmia was $10 \%$ in Group 1 and $<5 \%$ in other groups.

Most of the patients in all groups had applied to the hospital in 6-12 days after the onset of symptoms ( $63 \%$ on average for all groups).

\section{Chest computed tomography findings based on age}

The "normal lung CT findings" was the most common chest CT finding at the time of presentation to the hospital, except Group 2. This was followed by GGOs and GGOs with consolidation with the percentage of 31 and $22 \%$, respectively (Figure 1).
In all groups, GGOs tended to be in nodular/round shape, and it was statistically different in group $2(\mathrm{p}<0.05$; Table 1$)$.

The distribution of CT findings at the time of admission by groups is summarized in Table 2 .

GGOs with an ill-defined shape was encountered as often as the nodular form in Group $2(\mathrm{p}<0.05)$. However, whether GGO was nodular or ill-defined in shape, it was statistically significant to have a peripheral distribution in groups $1-3$ $(\mathrm{p}<0.05$; Table 1$)$.

The lesions were predominantly peripheral and subpleural in $659(49 \%)$ patients, and there was tendency to be in lower zone $(53 \%)$. In terms of peribronchovascular distribution of GGOs, there was no statistically difference between the groups ( $>0.05$; Table 1$)$.

The presence of consolidation without GGO in chest CT was extremely rare $(0.01 \%)$. The coexistence of GGO and consolidation with peripheral distribution in the lower lobes were common in all groups $(\mathrm{p}<0.05)$. If accompanying halo finding, the consolidation with GGO was statistically different in Group $2(\mathrm{p}<0.05)$ compared to other groups ( $>0.05)$ (Table 1).

In all groups, $134(10 \%)$ patients had focal pleural thickening. Of these, 115 were in the posterior pleura and 75 were adjacent to the lung lesion (Figure 2). This finding was statistically different for Groups 1-3 compared to other groups $(\mathrm{p}<0.05)$.

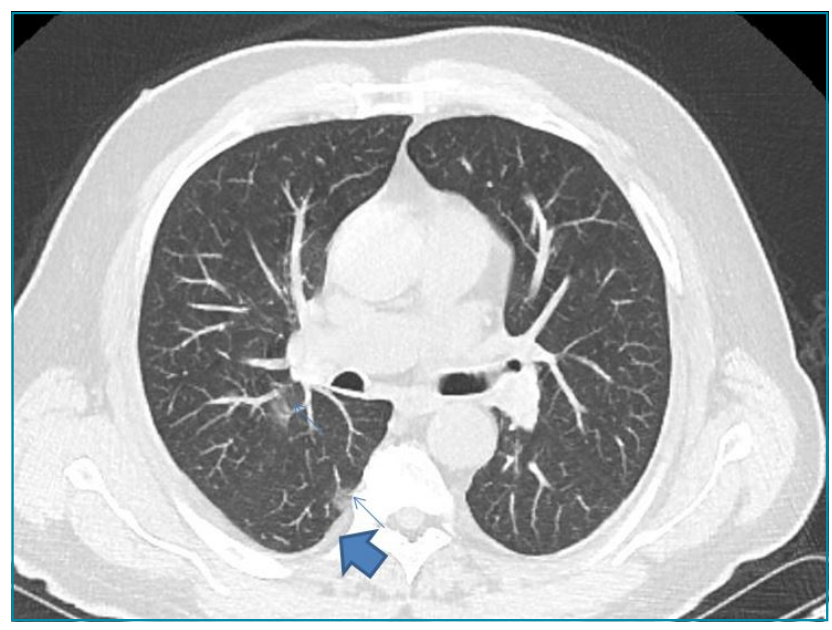

Figure 1. Graph showing the chest computed tomography findings of all patients at the time of presentation to the hospital. The most common chest computed tomography findings were normal. The presence of ground-glass opacity was the second most common lesion group, especially if it was bilateral. The cases with ground-glass opacity and consolidation were the third most common finding. The presence of consolidation alone was a very rare finding that the vast majority were due to atelectasis. 
Table 1. Chest computed tomography findings of ground-glass opacity in patients' groups.

\begin{tabular}{|c|c|c|c|c|c|c|}
\hline \multicolumn{2}{|r|}{ Range of } & $\begin{array}{c}\text { Group } 1 \\
18-30\end{array}$ & $\begin{array}{c}\text { Group } 2 \\
31-45\end{array}$ & $\begin{array}{c}\text { Group } 3 \\
46-55\end{array}$ & $\begin{array}{c}\text { Group } 4 \\
56-65\end{array}$ & $\underset{>65}{\text { Group }} 5$ \\
\hline & Number of patients & $(n=309)$ & $(n=434)$ & $(n=260)$ & $(n=119)$ & $(n=149)$ \\
\hline \multirow{22}{*}{$\begin{array}{l}\vec{Z} \\
\tilde{0} \\
0 \\
0 \\
\tilde{O} \\
\tilde{U} \\
\frac{0}{0} \\
\frac{1}{0} \\
\frac{0}{0} \\
\frac{0}{0}\end{array}$} & How the lung is involved? & & & & & \\
\hline & Upper lobes (n) & 81 & 28 & $103 *$ & 54 & 73 \\
\hline & Middle lobes (n) & 71 & 29 & $107^{*}$ & 57 & 69 \\
\hline & Lower lobes (n) & 141 * & 72 & $140 *$ & 70 & $103 *$ \\
\hline & Unilateral (n) & 61 & 64 & 30 & 12 & 15 \\
\hline & Bilateral (n) & 101 & 181 * & 120 & 66 & 96 \\
\hline & Unifocal (n) & 38 & 48 & 19 & 5 & 5 \\
\hline & Multifocal (n) & 118 & $197 *$ & 129 & 73 & 106 \\
\hline & Number of diseased segments (average \pm SD) & $7 \pm 5.2$ & $8.1 \pm 0.7$ & $8.5 \pm 2.1$ & $8.38 \pm 5.65$ & $9.16 \pm 1.4$ \\
\hline & Distribution feature of the lesion/lesions & & & & & \\
\hline & Central (n) & 31 & 27 & 17 & 4 & 2 \\
\hline & Peripheral (n) & $135^{*}$ & $205^{*}$ & $122^{*}$ & 68 & 94 \\
\hline & Peribronchovascular distribution ( $\mathrm{n}$ ) & 45 & 81 & 68 & 53 & 47 \\
\hline & Shape of lesion/lesions & & & & & \\
\hline & Nodular/round shape $(n)$ & 84 & $113^{*}$ & 59 & 36 & 45 \\
\hline & Lesions with irregular border (n) & 63 & $113^{*}$ & 75 & 41 & 66 \\
\hline & Coalescent lesions (n) & 19 & 37 & 28 & 19 & 27 \\
\hline & Accompanying $\mathrm{CT}$ findings & & & & & \\
\hline & Consolidation & 62 & $99 *$ & 47 & 34 & 41 \\
\hline & Halo sign & 35 & $86^{*}$ & 27 & 13 & 9 \\
\hline & Ters halo sign & 4 & 6 & 5 & 1 & 3 \\
\hline & Septal thickening & 50 & 63 & 52 & 34 & 64 \\
\hline
\end{tabular}

In our patient groups, the dominant lung lesion in chest computed tomography was ground-glass opacity. It tended to be bilateral in all groups. Groups 1-3 also had the feature of peripheral distribution in the lower lobes. In the presence of accompanying consolidation, the lower lobes and peripheral distribution were statistically significant, especially in Group 2. The presence of ground-glass density and halo finding to consolidation was statistically significant in Group 2. *Statistically significant at $p<0.05$. Since the findings described above can be seen together, the ratio has not been given. Statistical significance was specified.

Table 2. Distribution of the frequency of COVID-19 lung computed tomography findings by groups.

\begin{tabular}{l|c|c|c|c}
\hline Groups & $\begin{array}{c}\text { Negative for } \\
\text { pneumonia }\end{array}$ & $\begin{array}{c}\text { Typical appearance } \\
\text { for COVID-19 }\end{array}$ & $\begin{array}{c}\text { Indeterminate } \\
\text { appearance for COVID-19 }\end{array}$ & $\begin{array}{c}\text { Atypical appearance } \\
\text { for COVID-19 }\end{array}$ \\
\hline $\begin{array}{l}\text { Group 1 } \\
\mathrm{n}=309, \mathrm{n}(\%)\end{array}$ & $196(63)$ & $80(26)$ & $24(8)$ & $9(3)$ \\
\hline $\begin{array}{l}\text { Group 2 } \\
\mathrm{n}=434, \mathrm{n}(\%)\end{array}$ & $168(39)$ & $182(42)$ & $63(14)$ & $21(5)$ \\
\hline $\begin{array}{l}\text { Group 3 } \\
\mathrm{n}=260, \mathrm{n}(\%)\end{array}$ & $82(32)$ & $74(28)$ & $66(25)$ & $38(15)$ \\
\hline $\begin{array}{l}\text { Group 4 } \\
\mathrm{n}=119, \mathrm{n}(\%)\end{array}$ & $14(12)$ & $65(54)$ & $28(23)$ & $12(11)$ \\
\hline $\begin{array}{l}\text { Group 5 } \\
\mathrm{n}=149, \mathrm{n}(\%)\end{array}$ & $12(8)$ & $42(28)$ & $46(31)$ & $49(33)$ \\
\hline
\end{tabular}

In the Group 1, the frequency of normal lung findings (negative for pneumonia) is remarkable. The frequency of normal Chest computed findings in Groups 2 and 3 is remarkable as typical appearance for COVID-19. When all groups are examined, it is observed that the most common lung Chest computed finding is "negative for pneumonia" at the time of first admission to the hospital. 


\section{Chest computed tomography findings based on the duration of symptoms}

There were 786 chest CTs assessed in accordance with the early phase (P1). Normal chest CT findings were found in 404 patients in $\mathrm{P} 1$.

There were 310 patients evaluated as advanced-early phase (P2). Of these patients, 248 had chest CT findings typical of COVID-19.

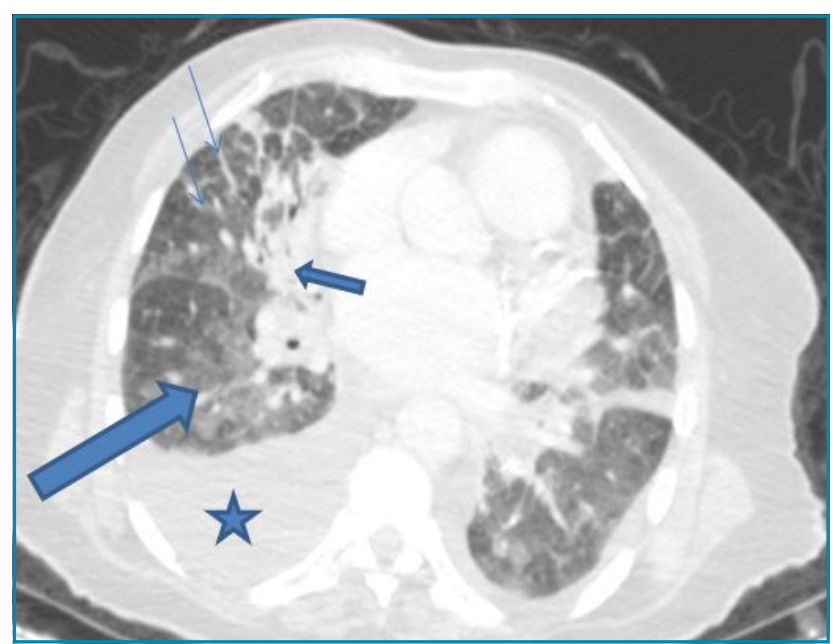

Figure 2. A 52-year-old male has been suffering from weakness and cough for about five days. There are groundglass opacity adjacent to the fissure and subpleural area (thin blue arrows). Focal pleural thickening is noticed in the right lower lobe adjacent to the lesion (thick blue arrow).
Typical chest CT findings for COVID-19 in the peak phase (P3) were $83 \%$.

There were 19 patients in the resolution phase (P4) in the study population. Typical chest CT findings for COVID-19 were the most common findings in $\mathrm{P} 4$.

There were no patients in recovery phase (P5) in our study population.

Of the 630 patients with typical findings, 343 were males and 287 were females. In patients with typical CT findings, the most common symptom was cough $(52 \%)$, followed by fever $(26 \%)$ and dyspnea (23\%).

Twenty-seven patients with pulmonary congestion findings were also clinically confirmed (Figure 3).

\section{Chest computed tomography findings based on the gender}

There was no statistically significant difference between men and women for any finding ( $p>0.05)$.

\section{DISCUSSION}

COVID-19 is the disease caused by a new subtype whose genetic structure is $82 \%$ similar to SARS-CoV ${ }^{1}$. The source of infection is wild animals and it can be transmitted through droplets or contact with a rapid infection, thus posing a huge threat to the public health ${ }^{1,3,12}$. Therefore, detecting the disease

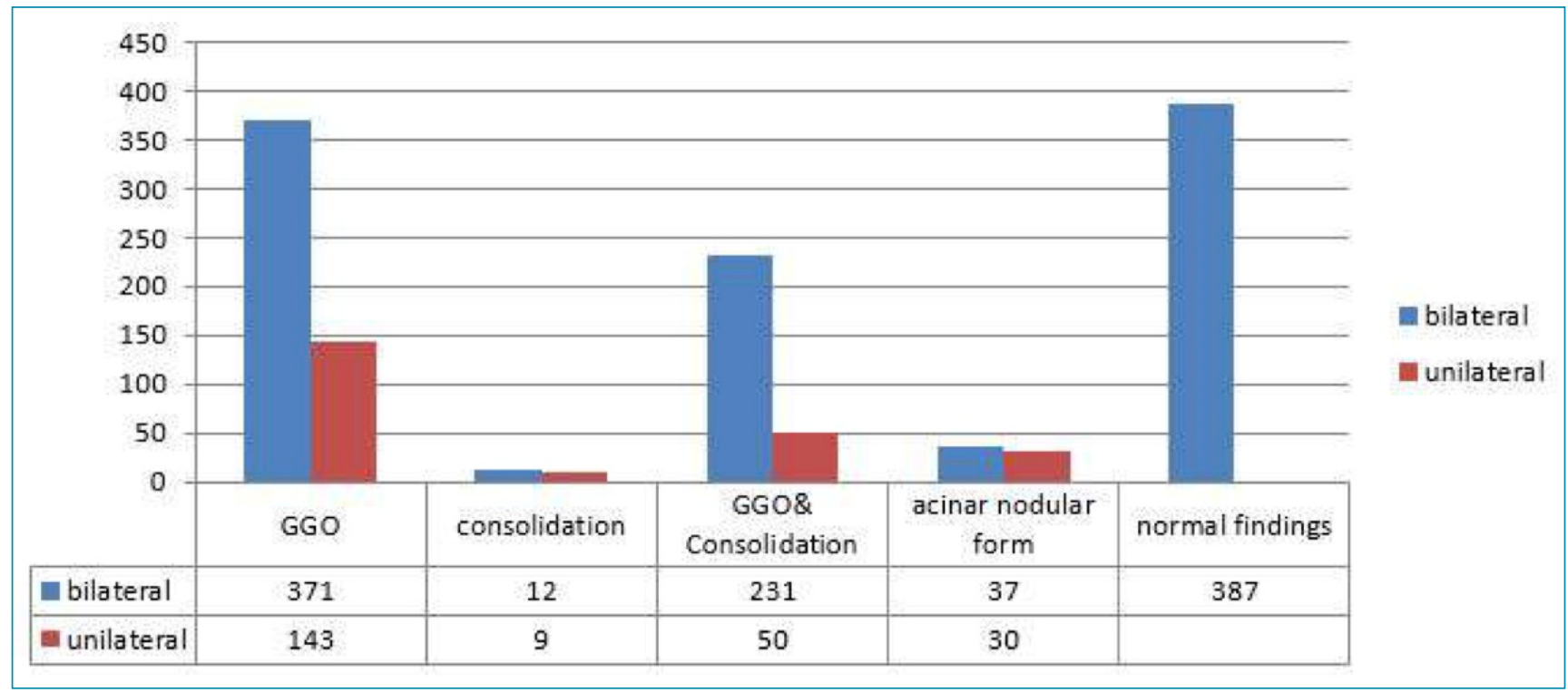

Figure 3. GGO: ground-glass opacity. A 76-year-old female patient has wheezing for the past 15 days and severe abdominal pain for one week. There are bilateral pleural effusion and cardiomegaly (blue stars). Bilaterally moderate interlobular septal thickening is observed (thin blue arrows). There is a focal consolidation in the right middle lobe (small thick blue arrow). Perihilar ground-glass opacity are present (long bold blue arrow). Chest computed tomography findings were classified as atypical findings for COVID-19. Pulmonary congestion findings in chest computed tomography suppress typical radiological findings for COVID-19. 
accurately and quickly should be the target ${ }^{12}$. As mentioned in the literature, chest CT has an important role in early diagnosis and follow-up of the disease ${ }^{8,9}$.

In our study, we evaluated chest CT findings of patients diagnosed with COVID-19 by RT-PCR test. We also evaluated the findings at the time of presentation to the hospital and whether they change with age, as well as how the duration of the findings affected chest $\mathrm{CT}$ findings.

The imaging manifestations of COVID-19 are similar to common viral pneumonia, especially SARS pneumonia, but there are some specific imaging and distribution characteristics of lesions ${ }^{1,3,4}$.

Peripheral distribution of GGOs and multisegment involvement of GGOs in early phase were typical ${ }^{11,12}$. This situation varied depending on the age ${ }^{11,12}$. In our younger age groups, typical findings were not observed in the early phase.

Therefore, it is important that the first CT scan is not performed in the first and second days of symptoms.

For the lung CT findings to be seen for COVID-19 as positive, the time interval required is found to be $3-5$ days after the onset of complaints in our study. Before this period, the diagnostic sensitivity of lung CT for COVID-19 is low ${ }^{13}$.

MERS pneumonia appears on CT images as subpleural and basilar airspace lesions, with extensive GGO and consolidation ${ }^{1}$.

In our patient groups, this was found to be quite significant, especially in group $2(p<0.05)$. The reason for this may be the late presentation to the hospital in this age group, which was most commonly between 6 and 12 days ${ }^{13-15}$.

In our study, we also evaluated the pleura in terms of pleural thickening. In the first three Groups (Groups 1-3), pleural thickening posteriorly or adjacent to the lesions was significantly more common than other locations $(\mathrm{p}<0.05)$.

In the literature, vascular engorgement, pleural thickening, and subpleural lines were reported in patients with COVID-1 $9^{7-9}$. The presence of pleural thickening may be a guiding radiological finding in patients whose parenchymal lesions have not yet developed or in those with suspicious findings in our study.

In other studies in the literature, it was stated that the presence of pleural effusion is a finding in the advanced stage of COVID-19 pneumonia ${ }^{13-15}$. In our study, we observed pleural effusion mostly in the older age group with CMG and clinically pulmonary congestion (Groups 4 and 5). In our study, early chest CT findings were evaluated at the time of presentation to the hospital. There were no follow-up chest CTs in the study. Multicenter studies will be needed to confirm this ${ }^{14,15}$

It was, however, not clear whether it was due to COVID19 or pulmonary congestion, both clinically and radiologically.
The clinician was advised to repeat the control chest CT after the treatment to relieve pulmonary congestion. In patients with other known malignancies, the typical findings of COVID-19 were easily recognized.

In the elderly patient group, the first CT findings had more severe parenchymal involvement compared to the other age group. The reason for this was interpreted as the prevalence of comorbidity in the patient group and the fact that first admission to the hospital was delayed compared to other groups.

It is also interesting that the older patient group had more indeterminate and atypical CT findings. This could be due to the severity or comorbidities of the older patients.

This study has some limitations. First, patients with negative RT-PCR tests were not included. As a result, the sensitivity of the chest CT could not be evaluated. Second, there were no children in our population. Third, the patient evolution or outcome was not evaluated. The frequency of thromboembolism has been reported as high in COVID-19 ${ }^{13-16}$. However, in our study protocol, we performed chest CT scan without administration of intravenous (IV) contrast agent. Therefore, we could not evaluate our patients in this regard. If the patient's clinic is suspicious in terms of pulmonary thromboembolism, we performed chest CT by adding IV contrast. And, we detected only for the presence of additional lung diseases (COPD, ILD, etc.) and hence there was lack of sufficient data on hypertension, diabetes mellitus, and other metabolic diseases at the time of diagnosis. Finally, sudden onset of pandemic caused inadequate documentation in the early stages of the disease.

\section{CONCLUSIONS}

It is noteworthy that the typical lung CT findings for COVID19 occur on days 6 to 10 (P3) of the illness period. This applies to all age groups. In addition, the most common symptoms in these patients with typical CT findings were cough, fever, and dyspnea. Therefore, it may be appropriate to perform the first CT screening of COVID-19 after 6 days based on the start time of the complaints for all age groups. In this way, the frequency and number of unnecessary radiation exposure can be reduced.

\section{AUTHORS" CONTRIBUTIONS}

SSDB: Conceptualization, Data curation, Formal analysis. ZS: Data curation. ASO: Data curation. FK: Data curation. MTA: Data curation. LD: Data curation. AI: Data curation. collected data. LD: Writing - original draft, Writing - review \& editing. YB: Writing - original draft, Writing review \& editing. 


\section{REFERENCES}

1. Koo HJ, Lim S, Choe J, Choi SH, Sung H, Do KH. Radiographic and CT features of viral pneumonia. Radiographics. 2018;38(3):71939. https://doi.org/10.1148/rg.2018170048

2. Guan WJ, Ni ZY, Hu Y, Liang WH, Ou CQ, He JX, et al. Clinical characteristics of Coronavirus disease 2019 in China. N Engl J Med. 2020;382(18):1708-20. https://doi.org/10.1056/ NEJMoa2002032

3. Hui DS, Azhar E, Madani TA, Ntoumi F, Kock R, Dar O, et al. The continuing 2019-nCoV epidemic threat of novel coronaviruses to global health - the latest 2019 novel coronavirus outbreak in Wuhan, China. Int J Infect Dis. 2020;91:264-6. https://doi. org/10.1016/j.ijid.2020.01.009

4. Salehi S, Abedi A, Balakrishnan S, Gholamrezanezhad A. Coronavirus disease 2019 (COVID-19): a systematic review of imaging findings in 919 patients. AJR Am J Roentgenol. 2020;215(1):87-93. https://doi.org/10.2214/ AJR.20.23034

5. Lan L, Xu D, Ye G, Xia C, Wang S, Li Y, et al. Positive RT-PCR test results in patients recovered from COVID-19. JAMA. 2020;323(15):1502-3. https://doi.org/10.1001/ jama.2020.2783

6. Yang Y, Yang M, Shen C, Wang F, Yuan J, Li J, et al. Evaluating the accuracy of different respiratory specimens in the laboratory diagnosis and monitoring the viral shedding of 2019-nCoV infections. Innovation (NY). 2020;1(3):100061. https://doi. org/10.1016/j.xinn.2020.100061

7. Wang Y, Dong C, Hu Y, Li C, Ren Q, Zhang X, et al. Temporal changes of CT findings in 90 patients with COVID-19 pneumonia: a longitudinal study. Radiology. 2020;296(2):E5564. https://doi.org/10.1148/radiol.2020200843

8. Ai T, Yang Z, Hou H, Zhan C, Chen C, Lv W, et al. Correlation of chest CT and RT-PCR testing for Coronavirus disease 2019 (COVID-19) in China: a report of 1014 cases. Radiology. 2020;296(2):E32-40. https://doi.org/10.1148/ radiol.2020200642
9. Chung M, Bernheim A, Mei X, Zhang N, Huang M, Zeng X, et al. CT imaging features of 2019 novel Coronavirus (2019nCoV). Radiology. 2020;295(1):202-7. https://doi.org/10.1148/ radiol.2020200230

10. Simpson S, Kay FU, Abbara S, Bhalla S, Chung JH, Chung M, et al Radiological Society of North America Expert consensus statement on reporting chest $C T$ findings related to COVID-19. endorsed by the society of thoracic radiology, the American College of Radiology, and RSNA - secondary publication. J Thorac Imaging. 2020;35(4):21927. https://doi.org/10.1097/RTI.0000000000000524

11. Dai WC, Zhang HW, Yu J, Xu HJ, Chen H, Luo SP, et al. CT imaging and differential diagnosis of COVID-19. Can Assoc Radiol J. 2020;71(2):195-200. https://doi. org/10.1177/0846537120913033

12. Chen N, Zhou M, Dong X, Qu J, Gong F, Han Y, et al. Epidemiological and clinical characteristics of 99 cases of 2019 novel coronavirus pneumonia in Wuhan, China: a descriptive study. Lancet. 2020;395(10223):507-13. https:// doi.org/10.1016/S0140-6736(20)30211-7

13. Huang $C$, Wang $Y$, Li X, Ren L, Zhao J, Hu Y, et al. Clinical features of patients infected with 2019 novel coronavirus in Wuhan, China. Lancet. 2020;395(10223):497-506. https:// doi.org/10.1016/S0140-6736(20)30183-5

14. Li Y, Xia L. Coronavirus Disease 2019 (COVID-19): role of chest $C T$ in diagnosis and management. AJR Am J Roentgenol. 2020;214(6):1280-6. https://doi.org/10.2214/AJR.20.22954

15. Pan $Y$, Guan $H$, Zhou S, Wang Y, Li Q, Zhu T, et al. Initial CT findings and temporal changes in patients with the novel coronavirus pneumonia (2019-nCoV): a study of 63 patients in Wuhan, China. Eur Radiol. 2020;30(6):3306-9. https://doi. org/10.1007/s00330-020-06731-x

16. Shi H, Han X, Jiang N, Cao Y, Alwalid O, Gu J, et al. Radiological findings from 81 patients with COVID-19 pneumonia in Wuhan, China: a descriptive study. Lancet Infect Dis. 2020;20(4):42534. https://doi.org/10.1016/S1473-3099(20)30086-4 


\section{ERRATUM}

\section{https://doi.org/10.1590/1806-9282.20210150ERRATUM}

In the manuscript "Chest computed tomography findings of 1271 patients with COVID-19 pneumonia and classifications with different age groups: a descriptive study from Istanbul, Turkey”, DOI: 10.1590/1806-9282.20210150, published in the Rev Assoc Med Bras. 2021;67(11):1531-1537:

\section{Page 1533, figure 1}

\section{Where it reads:}

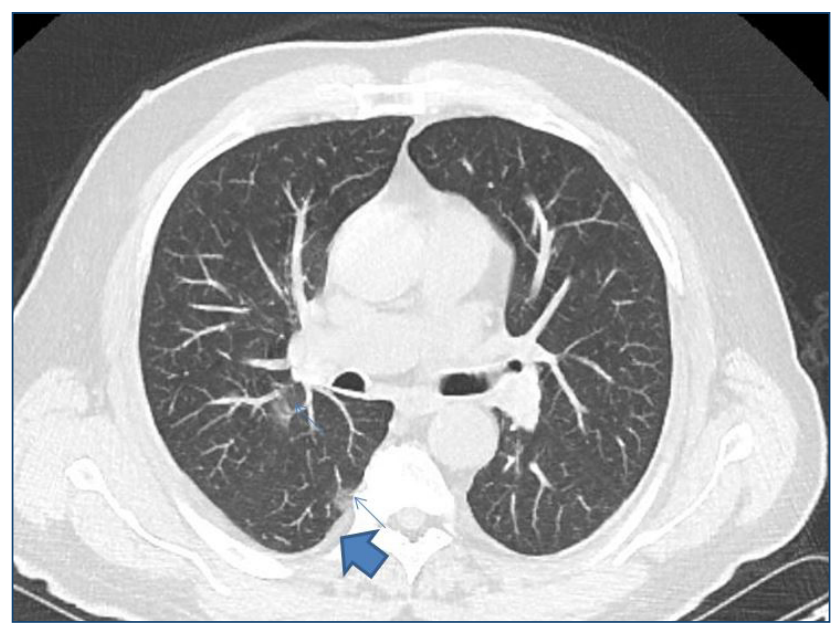

Figure 1. Graph showing the chest computed tomography findings of all patients at the time of presentation to the hospital. The most common chest computed tomography findings were normal. The presence of ground-glass opacity was the second most common lesion group, especially if it was bilateral. The cases with ground-glass opacity and consolidation were the third most common finding. The presence of consolidation alone was a very rare finding that the vast majority were due to atelectasis.

\section{Page 1535, figure 2}

\section{Where it reads:}

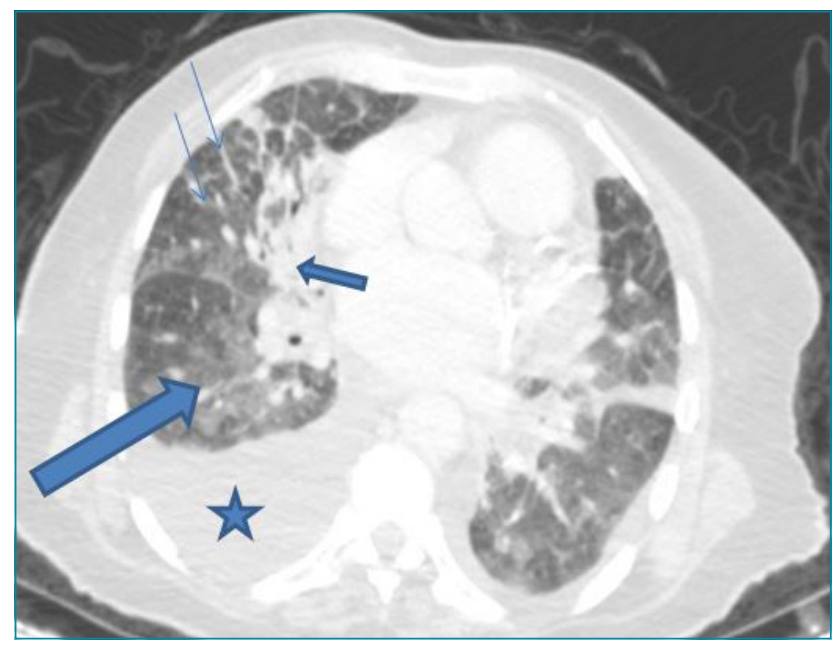

Figure 2. A 52-year-old male has been suffering from weakness and cough for about five days. There are groundglass opacity adjacent to the fissure and subpleural area (thin blue arrows). Focal pleural thickening is noticed in the right lower lobe adjacent to the lesion (thick blue arrow).

\section{It should read:}

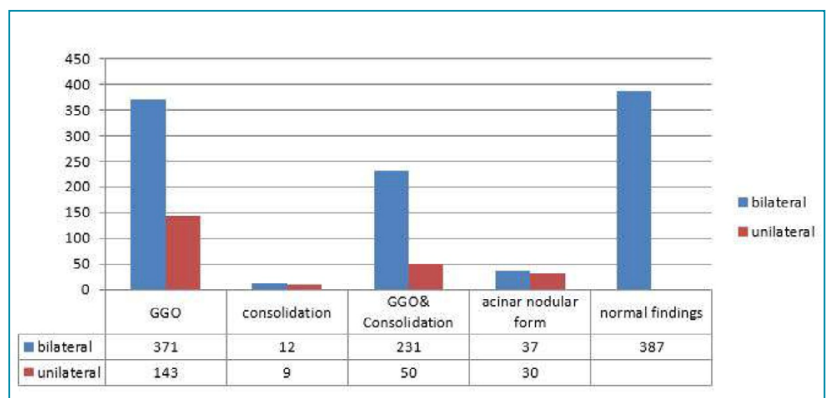

Figure 1. Graph showing the chest computed tomography findings of all patients at the time of presentation to the hospital. The most common chest computed tomography findings were normal. The presence of ground-glass opacity was the second most common lesion group, especially if it was bilateral. The cases with ground-glass opacity and consolidation were the third most common finding. The presence of consolidation alone was a very rare finding that the vast majority were due to atelectasis. (GGO: groundglass opacity).

\section{It should read:}

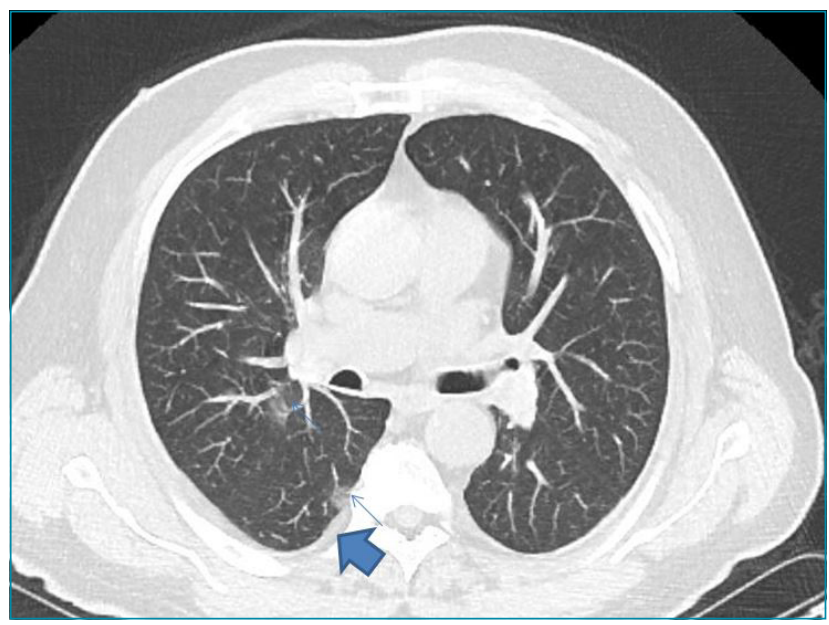

Figure 2. A 52-year-old male has been suffering from weakness and cough for about five days. There is groundglass opacity adjacent to the fissure and subpleural area (thin blue arrows). Focal pleural thickening is noticed in the right lower lobe adjacent to the lesion (thick blue arrow). 
Page 1535, figure 3

\section{Where it reads:}

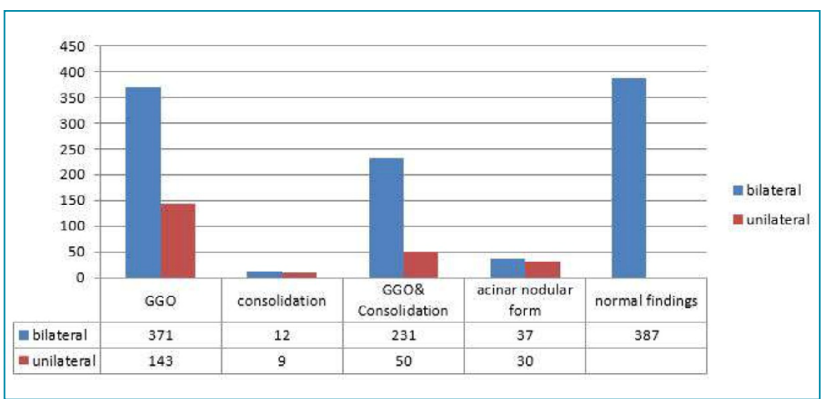

Figure 3. GGO: ground-glass opacity. A 76-year-old female patient has wheezing for the past 15 days and severe abdominal pain for one week. There are bilateral pleural effusion and cardiomegaly (blue stars). Bilaterally moderate interlobular septal thickening is observed (thin blue arrows). There is a focal consolidation in the right middle lobe (small thick blue arrow). Perihilar ground-glass opacity are present (long bold blue arrow). Chest computed tomography findings were classified as atypical findings for COVID-19. Pulmonary congestion findings in chest computed tomography suppress typical radiological findings for COVID-19.

\section{It should read:}

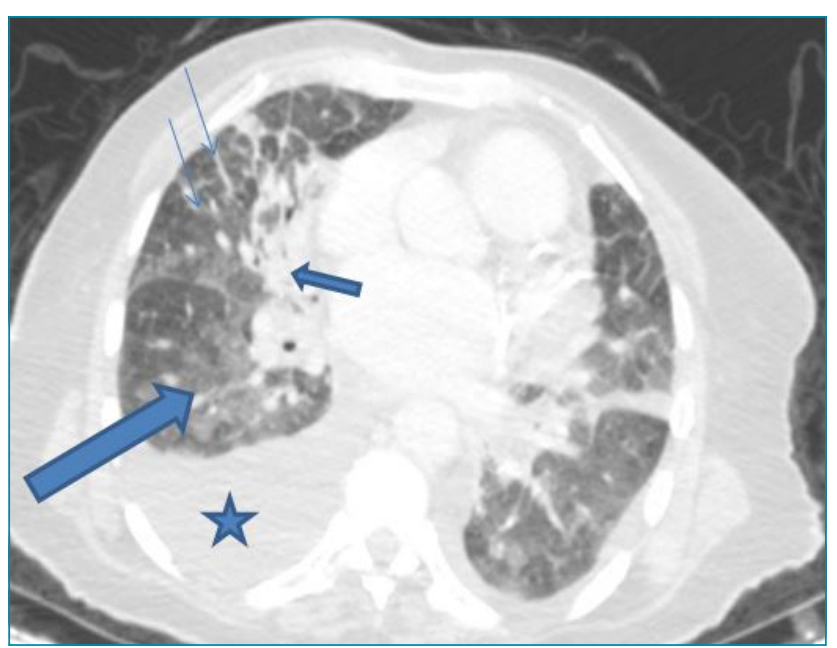

Figure 3. A 76-year-old female patient has wheezing for the past 15 days and severe abdominal pain for one week. There are bilateral pleural effusion and cardiomegaly (blue stars). Bilaterally moderate interlobular septal thickening is observed (thin blue arrows). There is a focal consolidation in the right middle lobe (small thick blue arrow). Perihilar ground-glass opacity are present (long bold blue arrow). Chest computed tomography findings were classified as atypical findings for COVID-19. Pulmonary congestion findings in chest computed tomography suppress typical radiological findings for COVID-19. 